International Mathematical Forum, 1, 2006, no. 39, 1913-1918

\title{
A Nonlocal Problem Arising from a Poiseuille Flow with Electrical Body Forces
}

\author{
Giovanni Cimatti \\ Department of Mathematics, University of Pisa \\ Largo Bruno Pontecorvo 5 \\ Pisa, 56127, Italy \\ cimatti@dm.unipi.it \\ Giovanni F. Gronchi \\ Department of Mathematics, University of Pisa \\ Largo Bruno Pontecorvo 5 \\ Pisa, 56127, Italy \\ gronchi@dm.unipi.it
}

\begin{abstract}
An approximation of the electric current density for the Poiseuille flow of a viscous slightly conducting fluid in a pipe of arbitrary cross section, in which a constant charge density is prescribed at one end and a difference of potential is applied, gives rise to a nonlocal elliptic problem which presents phenomena of bifurcation. The existence and multiplicity of solutions is studied.
\end{abstract}

Mathematics Subject Classification: 76W05, 35Q35, 76T20

Keywords: Poiseuille flow, nonlocal problem, bifurcation, multiplicity of solutions

In this paper we study the effect of body forces of electrical origin on the Poiseuille flow of a viscous, slightly conducting liquid (e.g. corn oil) in a straight pipe of finite length $L$ and arbitrary cross section $\Omega$, an open and bounded subset of $\mathbf{R}^{2}$ with a regular boundary $\Gamma$. We denote with $\Omega_{1}$ and $\Omega_{2}$ respectively the left and right end cross-section of the pipe and with $S$ the lateral surface of the tube. Let $\boldsymbol{\kappa}$ be the unit vector of the z-axis of the pipe and $(x, y)$ the coordinates in $\Omega$. We suppose, as in the theory of the Poiseuille flow, [3], [4]

$$
\mathbf{v}=v(x, y) \boldsymbol{\kappa}, \quad p=p(z)
$$


where $\mathbf{v}$ and $p$ are the velocity and pressure of the flow. Let $\varphi$ be the electric potential related to the electric field $\mathbf{E}$ by $\mathbf{E}=-\nabla \varphi$. We treat the case in which a constant difference of potential is applied between $\Omega_{1}$ and $\Omega_{2}$ :

$$
\varphi=0 \text { on } \Omega_{1}, \varphi=V \text { on } \Omega_{2}
$$

and the lateral surface $S$ of the pipe is electrically insulated, i.e.

$$
\frac{\partial \varphi}{\partial n}=0 \text { on } S
$$

Moreover, we assume that an injection of charge takes place on $\Omega_{1}$. Denoting $q$ the charge density, we have, with $q_{0}$ a given constant,

$$
q=q_{0} \text { on } \Omega_{1} \text {. }
$$

Neglecting diffusion, the current density $\mathbf{J}$ for a slightly conducting liquid, can be assumed [2], [5] to be given by $\mathbf{J}=\sigma \mathbf{E}+q \mathbf{v}$ where $\sigma$ is the (constant) electrical conductivity. In view of the boundary conditions (1), (2) and (3) and of the geometry of the problem, we treat the pipe as a straight one-dimensional body and therefore we assume, for the electrical part, $\mathbf{E}=E(z) \boldsymbol{\kappa}$ and $q=q(z)$. Thus we approximate the current density with the formula

$$
\mathbf{J}=J(z) \boldsymbol{\kappa}=(\sigma E(z)+q(z) \bar{v}) \boldsymbol{\kappa}
$$

where

$$
\bar{v}=\frac{1}{|\Omega|} \int_{\Omega} v(x, y) d x d y .
$$

The main advantage of this simplification somewhat unusual, since it is partly one dimensional and partly two-dimensional, is the possibility of determining $v(x, y)$ with a nonlocal boundary value problem (see e.g. [1]), which can be completely studied with respect to existence and number of solutions. Clearly we hope that these results can give valuable information for the full problem.

Since the flow is stationary, we have

$$
\frac{d J}{d z}=0
$$

Moreover Poisson's equation reduces to

$$
-\epsilon \frac{d^{2} \varphi}{d z^{2}}=q(z)
$$

where $\epsilon$ is the electrical permittivity. The corresponding boundary conditions are

$$
q(0)=q_{0}
$$




$$
\varphi(0)=0, \varphi(L)=V
$$

From (4)-(6) we get

$$
\bar{v} \frac{d q}{d z}(z)+\frac{\sigma}{\epsilon} q(z)=0
$$

which, together with (7), gives

$$
q(z)=q_{0} e^{-\frac{\sigma}{\epsilon \bar{v}} z}
$$

From Eqs. (6)-(9) we obtain

$$
\varphi(z)=\frac{q_{0} \epsilon \bar{v}^{2}}{L \sigma^{2}}\left(z e^{\frac{-\sigma L}{\epsilon \bar{v}}}-L e^{\frac{-\sigma z}{\epsilon \bar{v}}}+L-z\right)+\frac{V}{L} z .
$$

Body forces of electric origin are given by $q E$ and can be computed from Eqs. (9) and (10). Thus the Navier-Stokes system reduces to the single equation

$$
-\mu \Delta v(x, y)=-\frac{d p}{d z}(z)+q(z) E(z)
$$

where $\mu$ is the viscosity and

$$
q(z) E(z)=q_{0} e^{-\frac{\sigma}{\epsilon \bar{v}} z}\left[\frac{q_{0} \epsilon \bar{v}^{2}}{L \sigma^{2}}\left(1-e^{-\frac{\sigma L}{\epsilon \bar{v}}}-\frac{L \sigma}{\epsilon \bar{v}} e^{-\frac{\sigma z}{\epsilon \bar{v}}}\right)-\frac{V}{L}\right]
$$

As in the classical Poiseuille solution, we assume $p(0)-p(L)$ to be a given constant. Integrating Eq. $(11)$ over $(0, L)$ with respect to $z$ we obtain the nonlocal elliptic boundary value problem

$$
\left\{\begin{aligned}
-\Delta u & =\tilde{\lambda}+\tilde{a} f_{1}(\bar{u})+\tilde{b} f_{2}(\bar{u}) \\
u & =0, \text { on } \Gamma
\end{aligned}\right.
$$

where

$$
\begin{gathered}
u=\frac{\epsilon}{L \sigma} v, \quad \bar{u}=\frac{\epsilon}{L \sigma} \bar{v}, \quad \tilde{\lambda}=\frac{\epsilon(p(0)-p(L))}{L^{2} \mu \sigma}, \quad \tilde{b}=\frac{q_{0}^{2}}{\mu \sigma}, \quad \tilde{a}=\frac{q_{0} V \epsilon}{\mu L^{2} \sigma} \\
f_{1}(\xi)=\xi\left(e^{-\frac{1}{\xi}}-1\right), \quad f_{2}(\xi)=\frac{\xi^{2}}{2}\left(e^{-\frac{2}{\xi}}-1\right)+\xi^{3}\left(e^{-\frac{1}{\xi}}-1\right)^{2} .
\end{gathered}
$$

When $q_{0}=0$, we have the usual Poiseuille solution; thus hereafter we assume $q_{0} \neq 0$. To discuss the existence and number of solutions of the nonlocal problem $(12)$, let $w(x, y)$ be given by

$$
-\Delta w=1, w=0 \text { on } \Gamma
$$

and define $k=\bar{w}=\frac{1}{|\Omega|} \int_{\Omega} w(x, y) d x d y$. The constant $k$, positive by the maximum principle, contains all the information on the geometry of $\Omega$ which is 
needed to study problem (12). Let $\eta \in \mathbf{R}$ and $u=u(x, y ; \eta)$ be the solution of problem

$$
-\Delta u=\eta, u=0 \text { on } \Gamma \text {. }
$$

By uniqueness we have $u=\eta w$. Suppose now $\eta$ to be the unknown constant which appears in the right hand side of the differential equation in (12). Since $\bar{u}=\eta \bar{w}$, if $u$ is a solution to (12), the corresponding $\eta$ given by (13) satisfies the equation

$$
\eta=\tilde{\lambda}+\tilde{a} f_{1}(\eta k)+\tilde{b} f_{2}(\eta k)
$$

and, vice versa, to every solution of Eq. (14) there corresponds one and only one solution of (12). Setting

$$
\xi=k \eta, \quad \lambda=\tilde{\lambda} k, \quad a=\tilde{a} k, \quad b=\tilde{b} k,
$$

(14) is rewritten as

$$
g(\xi, a, b)=\lambda
$$

where

$$
g(\xi, a, b)=\xi-a f_{1}(\xi)-b f_{2}(\xi) .
$$

We note that $b$ is positive and $a \neq 0$. On the other hand, the sign of $\lambda$ depends on that of $p(0)-p(L)$. Studying the behavior of the function $g(\xi, a, b)$ for $a \neq 0$ and $b>0$ we find

$$
\begin{array}{ll}
\lim _{\xi \rightarrow-\infty} g(\xi, a, b)=-\infty, & \lim _{\xi \rightarrow 0-} g(\xi, a, b)=-\infty \\
\lim _{\xi \rightarrow 0+} g(\xi, a, b)=0, & \lim _{\xi \rightarrow \infty} g(\xi, a, b)=\infty
\end{array}
$$

Considering also the derivatives of $g(\xi, a, b)$ we arrive at the following conclusions:

(i) if $\lambda>0$ equation (15) and problem (12), generically have either one or three solutions;

(ii) if $\lambda<0$ equation (15) and problem (12) generically have either no solution or exactly two solutions. The three possible bifurcation diagrams in the $\lambda, \xi$ plane are shown in Figure 1: on the top we draw the bifurcation diagram for

$a=10^{-3}, b=10^{3}$. In the middle we use $a=-1 / 10, b=1$ while at the bottom we use $a=1, b=1$.

\section{References}

[1] M. Chipot and A. Rougirel, On some class of problems with nonlocal source and boundary flux, Adv. Differential Equations, 6 (2001), 1025 1048 . 

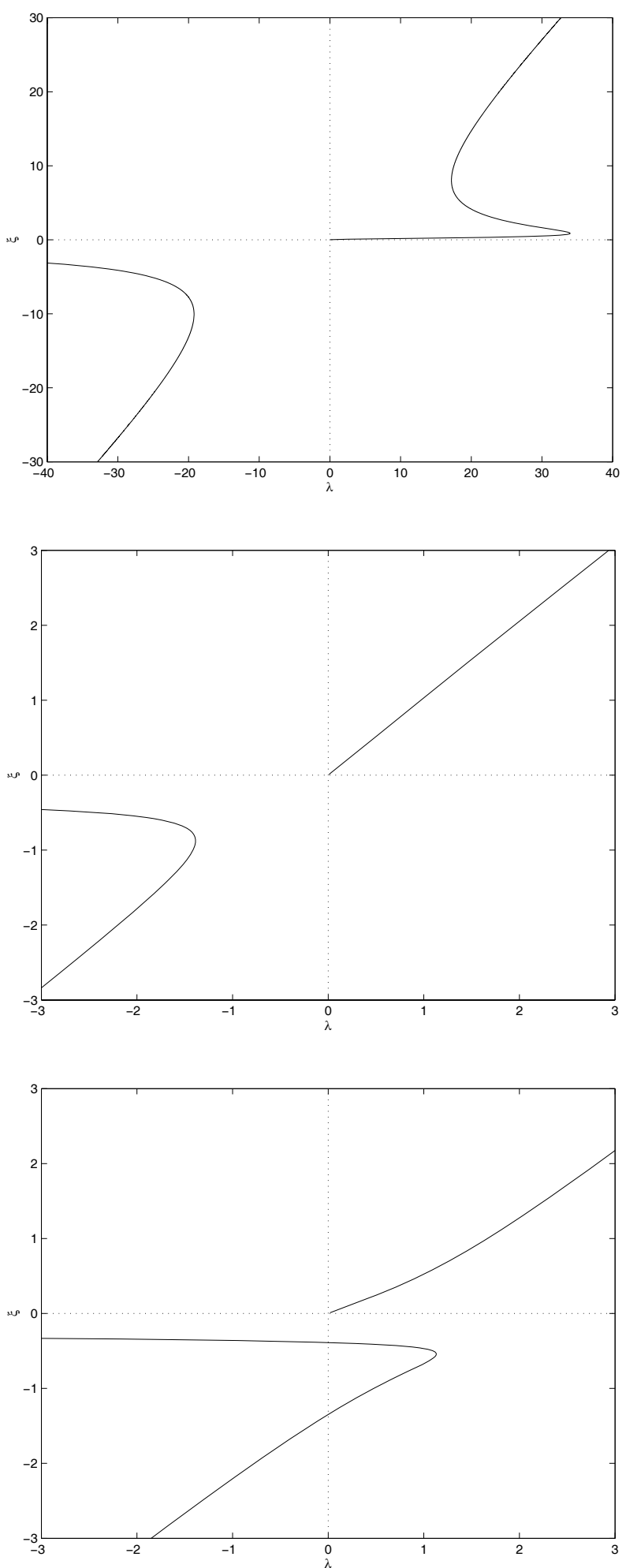

Figure 1: The three possible bifurcation diagrams of this problem are drawn with continuous lines in the $\lambda, \xi$ plane. 
[2] B.J. Deo and J.S. Richardson, Generalized energy method in electrohydrodynamics stability theory, J. Fluid Mech., 137 (1983), 131 - 151.

[3] H. Lamb, Hydrodynamics, Cambridge Univ. Press, 1975.

[4] G. Poiseuille, Recherches expèrimentales sur le mouvement des liquides dans le tubes de très petites diamètres, Comptes Rendus, XI (1840).

[5] B. Straughan, The Energy Method, Stability and Nonlinear Convection, Appl. Math. Sci. Ser., Springer, Berlin, 1992.

Received: May 4, 2006 